\title{
Bufferness phenomenon in distributed oscillatory systems
}

\author{
Nikolai Rozov
}




\title{
BUFFERNESS PHENOMENON IN DISTRIBUTED OSCILLATORY SYSTEMS
}

\author{
NikOLAI ROZOV \\ Moscow State University, Mechanical and Mathematical Department \\ 119899 MOSCOW, Russia \\ rozov@rozov.mccme.ru
}

[Received May 22, 2000]

\begin{abstract}
A theoretical approach to the investigation of the bufferness phenomenon in systems of differential equations is presented.

Mathematical Subject Classification: 35B10, 35B20

Keywords: Partial differential equations, singular perturbation, periodic solution, bufferness, oscillation
\end{abstract}

\section{Introduction}

It is widely known that the stable limit cycles of the ordinary differential equations play an exclusive role in the analysis of mathematical models of various oscillatory systems with lumped parameters (in physics, biology, economics, engineering etc.). However, it is useful to remember that the concept of a limit cycle was introduced by the great scientist, A. Poincaré, purely theoretically, in the early 80s of the 19th century. And only in the late 1920s did the outstanding Soviet physicist, A. A. Andronov, discovere that a stable limit cycle gives an adequate mathematical description of a steady periodic regime.

There are models where the matter of principle is the existence of unique stable limit cycle. In other models, however, it is interesting to discover the multiplicity of such cycles. Everything depends on the properties of concrete applied problem under consideration. Moreover, it is clear that, generally speaking, there exist various number of stable limit cycles, depending on the values of parameters in the system of differential equations. (These parameters describe actual properties of the object under investigation.)

It is possible to give examples of systems of ordinary differential equations, where, with appropriate choice of parameter values, one can guarantee the existence of an arbitrary, beforehand prescribed finite number of stable limit cycles. For example, let us consider the following second order system (in polar coordinates)

$$
\dot{r}=r \sin (1 / \alpha r), \dot{\varphi}=1,
$$

where $\alpha, 0<\alpha<1 / \pi$, is a parameter. For any natural number $N$ it is easy to indicate the value of parameter $\alpha$, positive and close to zero, providing exactly $N$ stable limit 
cycles, which are located in the area $r>1$ of phase plane.

As it was mentioned above, the mathematical models of oscillatory systems with lumped parameters are usually described by ordinary differential equations. However, nowadays in physics, chemistry, biology, new engineering and modern technologies the so-called oscillatory systems with distributed parameters or, more shortly, the distributed oscillatory systems are widely spread. These are objects, the state of which depends on both time and space variables; and the state of each point of space changes periodically with time. Thus, periodic auto-oscillations are generated, or auto-wave processes, following the term introduced by R. V. Khokhlov.

The dynamics of these objects is simulated, as a rule, by the partial differential equations (with boundary and initial conditions). Each actual auto-oscillatory regime corresponds to a stable cycle (it is a solution of the appropriate equation, periodic on time and satisfying the boundary conditions). The partial differential equation, being an adequate mathematical model of an oscillatory system with distributed parameters, can have one or several stable cycles. It is natural that the number of such cycles, generally speaking, may be different depending on the values of parameters in the equation.

It is said that the bufferness phenomenon takes place in the distributed oscillatory system if the system possesses the following property. The partial differential equation, which is the mathematical model of the system, possesses, under the appropriate values of parameters, an arbitrary, beforehand prescribed, finite number of different stable cycles. Theoretically speaking, for any natural number $N$ one can choose physical characteristics of the system so that it will have $N$ auto-oscillatory regimes.

The typical example of a distributed oscillatory system is the classical autogenerator containing a long line. The problem of investigation of its periodic (with time) regimes was initially formulated by A. A. Witt $[1,2]$. It is appropriate here to say more about this scientist, because his name is hardly known to mathematicians. Alexander A. Witt was one of the bright scientists representing the remarkable Soviet school of the oscillation theory (L. I. Mandelstam, N. D. Papalexi, A. A. Andronov, V. V. Migulin etc.). He carried out a number of interesting and perspective theoretical research. In particular, he was one of the co-authors of a classical book "The Oscillation Theory" [3]. However, as A. A. Witt was innocently convicted (and was killed in 1937), his name did not appear in the book [3] and was rehabilitated only after many years had passed $[4,5]$.

Let's point out that A. A. Witt proposed, in his work [2], the following hypothesis. There is a principal possibility that the oscillator with long line may possess several stable cycles simultaneously. (However, the bufferness phenomenon was not formulated yet). The fact that the number of auto-oscillating regimes may increase under the variation of the parameter values of the object was first observed during the physical experiment carried out by the research group of V. V. Migulin (see [6]). As for the mathematical investigation of the bufferness phenomenon, it began on Yu. S. Kolesov's initiative. He studied this phenomenon in parabolic reaction-diffusion 
systems [7] using numerical methods; later he completed the theoretical research for hyperbolic equations [8] (see also monograph [9]).

\section{Bufferness phenomenon for periodic solutions}

Let's consider one mathematical result which theoretically proves the existence of bufferness phenomenon in so-called $L C R G$-autogenerator composed on a long line with a tunnel diode. Omitting the description of physical details and the deduction of the necessary equations (it is standard enough), we consider the boundary value problem (see [10]) to be its mathematical model (under a number of conventional assumptions - in particular, under cubic approximation of the diode characteristics):

$$
\begin{aligned}
& u_{x}=-R i-L i_{t}, \quad i_{x}=-G u-C u_{t}, \\
& \left.i\right|_{x=1}=0,\left.\quad u\right|_{x=0}+\left.k_{0} u\right|_{x=1}+\left.k_{2} u^{2}\right|_{x=1}+\left.k_{3} u^{3}\right|_{x=1}=0,
\end{aligned}
$$

where $t$ is time, $x$ is the coordinate along a line, $u(t, x)$ and $i(t, x)$ are the voltage and the current in a line; all the autogenerator parameters (such as the distributed resistance $R$, inductance $L$, capacity $C$ and conductivity $G$ ) are constant. It is well known that this problem can be reduced to a boundary value problem for the so-called telegraph equation.

It is shown in [11] that the problem of searching for auto-oscillations in this model can be reformulated (after technical transforms and changes of variables) as a question of existence and stability of periodic solutions of a nonlinear differential-difference equation

$$
\begin{aligned}
& z^{\prime}(t)-(1-\alpha \epsilon) z^{\prime}(t-h)+(1-\epsilon) z(t)+(1-\alpha \epsilon)(1+\epsilon) z(t-h)= \\
& =a[z(t)-(1-\alpha \epsilon) z(t-h)]^{2}-[z(t)-(1-\alpha \epsilon) z(t-h)]^{3}
\end{aligned}
$$

here $\epsilon>0$ is a small parameter, the parameter $\alpha>0$ has the order of unit, the parameters $h=$ const $>0, a=$ const.

Let's denote by $\omega_{1}<\omega_{2}<\ldots \quad$ all the positive roots of the equation

$$
\omega \tan \frac{\omega h}{2}=1
$$

Lemma. All the roots of a quasi-polynomial

$$
P(\lambda)=\lambda\left[1-(1-\alpha \epsilon) e^{-\lambda h}\right]+(1-\epsilon)+(1-\alpha \epsilon)(1+\epsilon) e^{-\lambda h},
$$

corresponding to zero equilibrium state of equation (2), for $0<\epsilon \ll 1$ and $\alpha>$ $2\left(1+\omega_{1}^{2}\right)^{-1}$ lie in a half-plane Re $\lambda<0$. If the parameter $\alpha>0$ is decreasing, and when it becomes equal to the values $2\left(1+\omega_{n}^{2}\right)^{-1}, n \geq 1$, then the roots of a quasipolynomial $P(\lambda)$ transfer sequentially, one after another, to the half-plane Re $\lambda>0$.

Theorem. Let for some natural $n \geq 1$ the conditions

$$
0<\alpha<\frac{2}{1+2 \omega_{n}^{2}-\omega_{1}^{2}}, \quad 1+10 \omega_{n}^{2}-15 \omega_{n}^{4} \neq 0
$$


be fulfilled. Then there exists $\epsilon_{n}>0$, such that for any $\epsilon \in\left[0, \epsilon_{n}\right]$ the equation (2) has an exponentially orbital-stable (in metrics of the phase space $W_{2}^{1}(-h, 0)$ ) periodic solution $z_{n}(\tau, \epsilon)$ :

$$
z_{n}\left(\tau+\frac{2 \pi}{\omega_{n}}, \epsilon\right)=z_{n}(\tau, \epsilon), \quad \tau=\left(1+\delta_{n}(\epsilon)\right) t .
$$

For this solution the following asymptotic (when $\epsilon \rightarrow 0)$ representation is valid:

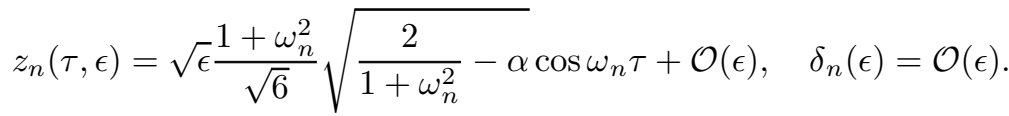

It follows from the above Theorem (the proof of which is rather nontrivial), that the following dynamics of equation (2) takes place under the appropriate decrease of the parameters $\epsilon$ and $\alpha$.

Corollary. Zero equilibrium state of equation (2) is exponentially stable for all $\alpha>2\left(1+\omega_{1}^{2}\right)^{-1}$. When the parameter $\alpha$ is equal to $2\left(1+\omega_{1}^{2}\right)^{-1}$, then a stable cycle branches from this equilibrium state (the Andronov - Hopf bifurcation). For the further decrease of $\alpha$, when it becomes equal to each value $2\left(1+\omega_{n}^{2}\right)^{-1}, n \geq 2$, an unstable cycle appears (the secondary Andronov - Hopf bifurcation), which becomes stable for $\alpha>2\left(1+2 \omega_{n}^{2}-\omega_{1}^{2}\right)^{-1}$.

Thus, under appropriate decrease of parameters $\epsilon$ and $\alpha$, one can guarantee the existence of any beforehand prescribed number of stable cycles for equation (2). It means that the bufferness phenomenon takes place for the system (1). That is, the mathematical model of the $L C R G$-autogenerator with a long line, under suitable choice of the values of its parameters, may possess an arbitrary number of stable periodic regimes.

Let's point out that the last conclusion can be illustrated by the real experiment (see $[12])$.

To summarize all the above information, let us formulate the specific features of the bufferness phenomenon from the physical point of view. A distributed oscillatory system possessing the bufferness phenomenon has a set of stable periodic regimes. Note that under the appropriate choice of the system parameters, this set may contain an arbitrary large number of such regimes. When the values of parameters are fixed, then the concrete regime, one of the potentially possible auto-oscillatory regimes, comes into realization depending on the initial conditions or external factors. The spontaneous transition of the system to some other periodic regime is impossible.

The further research concerning the bufferness phenomenon and associated problems is discussed in the monograph [13].

As a conclusion we will consider one more rather simple concrete example of a system with a bufferness property - an example from mechanics.

Let's consider an auto-oscillatory system consisting of the homogeneous string of the length $l$ with fixed ends, to the middle of which a generator of mechanical 
oscillations is connected. Let's assume that the oscillating string is characterized by density $\rho$, tension $T$ and condensation of friction forces $h$. As for the generator, it is represented by a resonator consisting of the mass $M$, a spring with the elasticity $k$ and nonlinear element of friction $h_{*}(v)=\lambda\left(v^{2}-1\right), \lambda>0$.

Omitting the rather simple deduction of the equations for the string and generator, we shall consider only the final mathematical model (after necessary normalization of the variables). It is the following boundary value problem in the segment $0 \leq x \leq 1$ :

$$
\begin{gathered}
u_{t t}+\varepsilon u_{t}=u_{x x}, \\
\left.u\right|_{x=0}=0,\left.\quad\left[u_{t t}+\varepsilon \alpha\left(u^{2}-1\right) u_{t}+\beta u\right]\right|_{x=1}=-\left.\gamma u_{x}\right|_{x=1} .
\end{gathered}
$$

Here the required function $u(t, x)$ characterizes the shift of an element of the string, $0<\varepsilon \ll 1$, and parameters $\alpha, \beta, \gamma>0$ have the order of unit.

It appears (under certain assumptions, which are not formulated here for brevty's sake), that, with the appropriate increase of parameter $\alpha$ and proper decrease of parameter $\varepsilon$, one can guarantee that this boundary value problem possesses an arbitrary beforehand prescribed finite number of stable solutions, periodic with $t$; i.e. the bufferness phenomenon is observed.

\section{REFERENCES}

[1] Witt A. A.: The distributed auto-oscillatory systems, Zhurn. Techn. Fiziki, 4(1), (1934), 144-157. (in Russian)

[2] Witt A. A.: To the theory of the violin string, Zhurn. Techn. Fiziki 6(9), (1936), 1459-1479. (in Russian)

[3] Andronov, A. A. and Khaikin, S. E.: The Oscillation Theory, Moscow, 1937. (in Russian)

[4] Andronov, A. A., Witt A. A. and Khaikin, S. E.: The Oscillation Theory, Recast and additions of N. A. Zheleztsov, Moscow, 1959. (in Russian)

[5] Andronov, A. A., Witt A. A. and Khaikin, S. E.: The oscillation theory, Moscow, 1981. (in Russian)

[6] Az'Yan, Yu. M. and Migulin, V. V.: On auto-oscillations in a system with delayed feedback, Radiotekhnika i electronika 1(4), (1956), 418-427. (in Russian)

[7] Zakharov, A. A. and Kolesov, Yu. S.: Spatially nonhomogeneous modes in the predator-victim problem, In: Nonlinear Oscillations and Ecology, Yaroslavl, 1984, 3-15. (in Russian)

[8] Kolesov, A. Yu. and Kolesov, Yu. S.: Bifurcation of auto-oscillations in singularly perturbed wave equation, Dokl. Akad. Nauk SSSR, 315(12),(1990), 281-283. (in Russian)

[9] Mishchenko, E. F., Kolesov, Yu. S., Kolesov, A. Yu. and Rozov, N. Kh.: Periodic Motion and Bifurcation Processes in Singularly Perturbed Systems, Moscow, 1995. (in Russian)

[10] Tikhonov, A. N. and Samarsky, A. A.: Equations of Mathematical Physics, Moscow, 1977. (in Russian) 
[11] Kolesov, Yu. S. and Shvitra, D. I.: Auto-oscillations in a long line with the tunnel diode, In: Differential equations and their applications, Vilnius, 1977, 27-48. (in Russian)

[12] Kambulov, V. F., Kolesov, A. Yu. and Rozov, N. Kн.: Theoretical and experimental analysis of the bufferness phenomenon in a long line with the tunnel diode, Differential equations, 33(5), (1997), 638-645. (in Russian)

[13] Kolesov, A. Yu., Mishchenko, E. F. and Rozov, N. Kh.: Asymptotic methods for investigation of periodic solutions in nonlinear hyperbolic equations, Moscow, 1998. (Trudy MIAN, volume 222.) (in Russian) 